\title{
CORRELATION BETWEEN HBA1C AND LIPID PROFILE IN PATIENTS WITH TYPE 2 DIABETES MELLITUS AT PIRNGADI HOSPITAL, MEDAN, NORTH SUMATERA
}

\author{
Endy Julianto'), Hendrika Andriana Silitonga'), \\ Jekson Martiar Siahaan3) \\ 1) Department of Parasitology, Faculty of Medicine, \\ Methodist University, Medan \\ 2) Department of Histology, Faculty of Medicine, \\ North Sumatera University, Medan \\ 3) Department of Physiology, Faculty of Medicine, \\ North Sumatera University, Medan
}

\begin{abstract}
Background: Diabetes Mellitus (type 2 DM) is now ranked first in health problems. The Diabetes Control and Complication Trial (DCCT) study showed that lowering HbA1c rates may delay or prevent chronic complications. One of the risk factors for CHD in type $2 \mathrm{DM}$ is dyslipidemia, i.e lipid metabolism disorders in the form of elevated total cholesterol, triglyceride (TG), low-density lipoprotein (LDL), and decreased levels of high-density lipoprotein (HDL). Description of dyslipidemia in type 2 DM most frequently found is an increase in TG levels and decreased HDL levels. Research showed hyperglycemic conditions have an effect on the condition of blood vessels. This study aimed to determine the correlation between HBA1C and lipid profile in patients with type 2 DM.
\end{abstract}

Subjects and Method: This was an analytic observational study with a crosssectional design. The study was conducted at the Endocrinology and Metabolic polyclinic Pirngadi Hospital, Medan, North Sumatera, from January to February 2018. A total sample of 10 type 2 diabetes mellitus was selected for this study. The independent variables were lipid profile including total cholesterol, high density lipoprotein (HDL), low density lipoprotein (LDL), and trygliceride. The dependent variable was HbA1c level. Lipid profile was measured by blood serum examination. The data of type 2 diabetes mellitus were taken from medical record. HbA1c level was measured by blood examination. The data were analyzed by Pearson correlation.

Results: HbA1c was positively correlated with blood sugar levels and was statistically significant $(\mathrm{r}=0.12 ; \mathrm{p}=0.005)$. HbA1c correlated weakly and was statistically insignificant with total cholesterol $(\mathrm{r}=0.14, \mathrm{p}=0.702)$, LDL $(\mathrm{r}=0.15$; $\mathrm{p}=0.683)$, HDL $(\mathrm{r}=0.04 ; \mathrm{p}=0.922)$, and triglycerides $(\mathrm{r}=0.06 ; \mathrm{p}=0.860)$.

Conclusion: HbA1c correlates with blood sugar levels but does not show correlation with lipid profiles.

Correspondence:

Endy Julianto. Department of Parasitology, Faculty of Medicine, Methodist University, Medan. 